\title{
Distribution and community structure of ichthyoplankton off the coast of Oregon, USA, in 2000 and 2002
}

\author{
Toby D. Auth ${ }^{1, *}$, Richard D. Brodeur ${ }^{2}$ \\ ${ }^{1}$ Cooperative Institute for Marine Resources Studies, Oregon State University, Newport, Oregon 97365, USA \\ ${ }^{2}$ Northwest Fisheries Science Center, NOAA Fisheries, Newport, Oregon 97365, USA
}

\begin{abstract}
The species composition, distribution and concentration of ichthyoplankton off the central Oregon coast in the NE Pacific Ocean were examined during 2000 and 2002 to investigate annual, seasonal, vertical, and cross-shelf variability. Larval concentrations were also analyzed in relation to water temperature and salinity. The 281 samples collected from 5 cruises along a historically sampled transect between April and September in each of the 2 study years yielded 4944 fish larvae comprising 72 taxa in 28 families. The dominant taxa collected were Engraulis mordax, Lyopsetta exilis, Sebastes spp., Stenobrachius leucopsarus and Tarletonbeania crenularis. Total larval concentration increased from 49.3 per $1000 \mathrm{~m}^{3}$ in 2000 to 72.0 per $1000 \mathrm{~m}^{3}$ in 2002, with seasonal concentrations highest in August 2000 (90.3 per $1000 \mathrm{~m}^{3}$ ) and April 2002 (151.2 per $1000 \mathrm{~m}^{3}$ ). Relatively few larvae were found at depths $>100 \mathrm{~m}$, while highest larval concentrations generally occurred from 0 to $50 \mathrm{~m}$. However, L. exilis concentrations were highest from 50 to $100 \mathrm{~m}$. Larval diversity and concentration were higher offshore (46 to $84 \mathrm{~km}$ ) than in coastal areas (9 to $28 \mathrm{~km}$ ). Highest concentrations were normally found at an intermediate station $65 \mathrm{~km}$ off the coast. Species designated as either coastal or offshore species by previous studies were predominantly found in their respective shelf regions. With the exception of $L$. exilis, larval concentrations were positively correlated with temperature and negatively correlated with salinity $(\mathrm{p}<0.0001)$.
\end{abstract}

KEY WORDS: Ichthyoplankton · Fish larvae · Assemblages · Distributions · Multivariate analyses North Pacific Ocean · Oregon coast

\section{INTRODUCTION}

Larval-stage dynamics can influence the trophic structure of marine communities (Hunter \& Kimbrell 1980, Young \& Davis 1990), and may be used as indicators of future recruitment potential in adult fish stocks (Hjort 1914, Houde 1997). Sherman et al. (1983) recognized the importance of larval studies to fisheries management, stating ' ...ichthyoplankton surveys represent the most effective sampling strategy available for measuring abundance levels of all fish species inhabiting the LMEs' (large marine ecosystems). However, the community structure and trophic function of larval fish populations as well as their response to environmental fluctuations and influence on adult fish stocks are poorly understood for many of the dominant taxa in the northern California Current LME. In response to this, the US GLOBEC (Global Ocean Ecosystems Dynamics) NE Pacific Program initiated the 'Long-Term Observation Program' (LTOP) in 1997 and the 'Mesoscale Dynamics Program' (MDP) in 2000 to examine the link between environmental change and factors that affect the recruitment of marine fish and invertebrate populations in the NE Pacific Ocean, including larval fish population structures (Batchelder et al. 2002). In the past decade, this region has experienced extreme and variable climate-induced events (e.g. regime shift, El Niño, La Niña, drought, nearshore hypoxia) (Schwing \& Moore 2000, Schwing et al. 2000, Smith \& Moser 2003, Grantham et al. 2004) that have afforded a unique opportunity to investigate the response of larval fish communities to dramatic environmental shifts in the region. 
The present study is the first from the LTOP and MDP to analyze larval fish population structures in the northern California Current. Although sampling occurred along much of the southern Oregon and part of the northern California coasts as part of the LTOP and MDP, for this study we chose to examine ichthyoplankton samples from a single transect off the central Oregon coast, known as the Newport Hydrographic (NH) line. Ichthyoplankton have been historically well sampled along this transect (Waldron 1972, Richardson 1973, Richardson \& Pearcy 1977, Richardson et al. 1980, Mundy 1984, Boehlert et al. 1985, Brodeur et al. 1985, Doyle 1992), allowing for the contrast and comparison of larval fish populations and assemblages in the same location over several decades. However, although sampled intermittently from 1967 (Waldron 1972) to 1987 (Doyle 1992), no ichthyoplankton have been analyzed from this region since 1987. Also, no previous study has examined the community structure of all fish larvae along the NH line across annual, seasonal, zonal (coastal vs. offshore) and depth-stratified scales.

The purpose of this study was to identify, quantify and compare larval fish distributions, concentrations and assemblages off the central Oregon coast between April and September in 2000 and 2002. We also tested for differences in larval fish population structures between coastal and offshore stations, and at discrete depth layers from the surface to $>100 \mathrm{~m}$ depth during each month sampled. Larval concentrations and distributions were analyzed to develop and compare diversity and evenness indices and species assemblages across the aforementioned spatial and temporal scales. Multivariate and correlation analyses were used to discern the possible influences of water temperature and salinity on these distributions. Through these analyses, we hoped to achieve a better understanding of recent larval fish population dynamics in the northern California Current, and compare them to those found by previous studies in the last few decades to determine what, if any, changes have occurred.

\section{MATERIALS AND METHODS}

Sampling procedures. Ichthyoplankton samples were collected from 5 stations spaced approximately $19 \mathrm{~km}$ apart along a single transect ( $\mathrm{NH}$ line) extending 9 to $84 \mathrm{~km}$ off the central Oregon coast (Fig. 1). Sampling was carried out during 5 cruises between April and September each in 2000 and 2002, resulting in the collection of 281 depth-stratified samples from 43 hauls (Table 1). Sampling was done at different times during both day and night. A multiple opening/closing net and environmental sensing system (MOCNESS; Wiebe

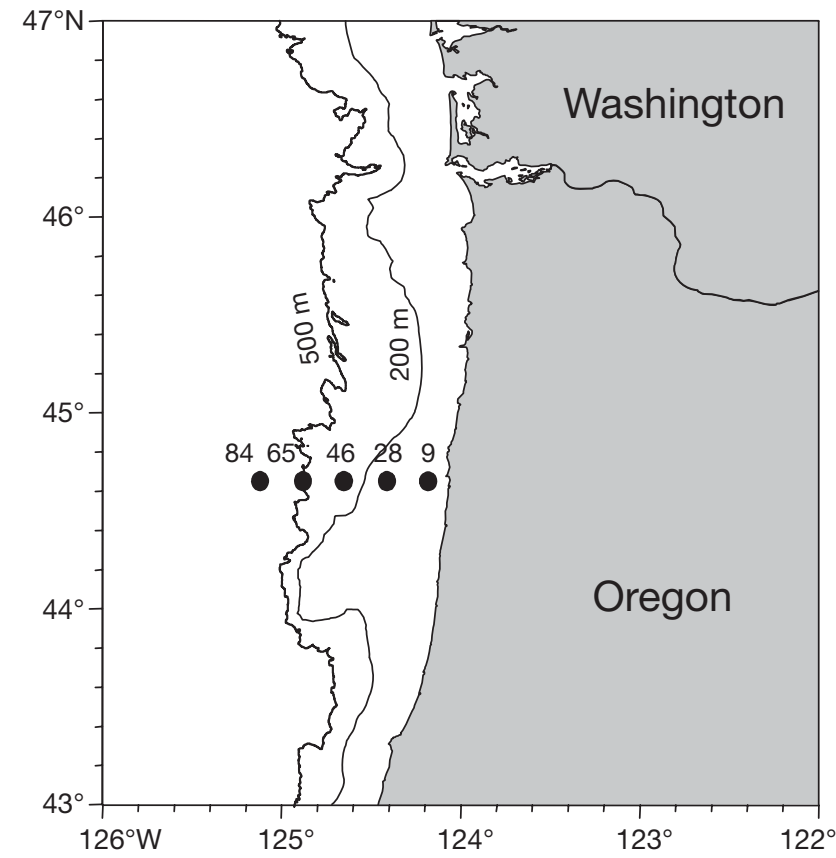

Fig. 1. Locations of stations sampled between April and September in 2000 and 2002. Depth contours of 200 and $500 \mathrm{~m}$ are shown

et al. 1976) with a $1 \mathrm{~m}^{2}$ mouth opening and $333 \mu \mathrm{m}$ mesh nets was used to collect ichthyoplankton at 3 to 9 discrete depths (typically $0-10,10-20,20-50,50-100$, $100-150,150-200,200-300$ and $>300 \mathrm{~m}$ when applicable, with substrata sampled during certain hauls). Volume filtered, temperature, salinity depth of the net, wire out, and angle of the net mouth relative to the geoid were recorded throughout the water column. The MOCNESS was fished as a continuous oblique tow from within 5 to $10 \mathrm{~m}$ of the bottom (or to a maximum depth of $350 \mathrm{~m}$ ) to the surface at a line retrieval rate of 20 to $30 \mathrm{~m} \mathrm{~min}^{-1}$ and a ship speed of 1 to $1.5 \mathrm{~m} \mathrm{~s}^{-1}$. Ship and retrieval speeds were continually adjusted during each tow so as to maintain a mouth opening of $45^{\circ}$. In so doing, we maintained an effective mouth opening of $1 \mathrm{~m}^{2}$ at all times. Mean water volume filtered by each net was $206 \mathrm{~m}^{3}(\mathrm{SE}=9.63)$.

Ichthyoplankton samples were preserved in a $10 \%$ buffered-formalin seawater solution at sea. Fish larvae from each sample were completely sorted, counted, and identified to the lowest taxonomic level possible in the laboratory using a dissecting microscope. The lesser of either all larvae or a random subsample of 50 individuals from each species in each sample were measured to the nearest $0.1 \mathrm{~mm}$ standard length (SL) using an ocular micrometer mounted on the sorting microscope.

Data analyses. Larval concentrations for each depthstratified sample $\left(D_{i}\right)$ were expressed as the number of individuals per $1000 \mathrm{~m}^{3}$. Weighted mean water-column 
Table 1. Number of depth-stratified ichthyoplankton samples collected at each station during each cruise off the Oregon coast. Boldface: samples collected at night; normal print: samples collected by day; -: not sampled

\begin{tabular}{|lccccc|}
\hline \multirow{2}{*}{ Cruise date } & \multicolumn{5}{c|}{ Station no. (km from shore) } \\
& 9 & 28 & 46 & 65 & 84 \\
\hline Apr 11-12, 2000 & $\mathbf{4}$ & $\mathbf{5}$ & $\mathbf{7}$ & 8 & 8 \\
Jun 3, 2000 & - & 5 & 8 & - & - \\
Jul 7-8, 2000 & 4 & 7 & $\mathbf{8}$ & $\mathbf{8}$ & $\mathbf{8}$ \\
Aug 4, 2000 & 5 & 5 & 6 & $\mathbf{7}$ & $\mathbf{7}$ \\
Sep 7-8, 2000 & 3 & 3 & $\mathbf{8}$ & $\mathbf{8}$ & $\mathbf{8}$ \\
Apr 4-5, 2002 & 3 & $\mathbf{4}$ & $\mathbf{8}$ & $\mathbf{9}$ & 9 \\
May 30, 2002 & - & $\mathbf{5}$ & $\mathbf{8}$ & 8 & 8 \\
Jul 9-10, 2002 & 3 & $\mathbf{5}$ & $\mathbf{8}$ & $\mathbf{8}$ & 8 \\
Aug 8-9, 2002 & - & $\mathbf{5}$ & $\mathbf{9}$ & - & - \\
Sep 27-28, 2002 & $\mathbf{4}$ & $\mathbf{5}$ & 8 & 8 & 8 \\
\hline
\end{tabular}

concentrations for each haul $\left(D_{\text {Haul }}\right)$ were calculated according to the equation:

$$
D_{\text {Haul }}=\sum D_{i} \cdot d_{i} / \sum d_{i}
$$

where $d_{i}$ is the depth range $(\mathrm{m})$ of each depth-stratified sample $i$. To facilitate vertical distribution analyses, the water column was divided into 5 depth strata: 0-10, $10-20,20-50,50-100$ and $>100 \mathrm{~m}$. Weighted mean concentrations for all samples collected in each depth stratum per haul were calculated as haul concentration. ANOVA and a Tukey's multiple range test were applied to the $\log _{e}(N+0.1)$-transformed haul and depth strata concentrations to test for significant differences between years, months, station locations and depth strata. Weighted mean (based on concentration) larval lengths of important species were also calculated for each haul and depth stratum, and similarly tested for significant differences between years, months, station locations and depth strata. Interaction terms were not included in the ANOVA model because of the limited sample size. All ANOVA were performed using JMP IN Version 5.1 statistical software (SAS Institute 2005).

Taxon diversity and evenness were analyzed based on all identifiable collected taxa $(\mathrm{n}=71)$ across annual, seasonal, coastal/offshore and depth-stratified scales. The Shannon-Wiener diversity index $\left(H^{\prime}\right)$ was used to measure larval diversity, where higher $H^{\prime}$ values denote greater diversity. Taxa evenness was assessed using Pielou's evenness index $\left(J^{\prime}\right)$, which ranges from 0 to 1 , with the maximum $J^{\prime}$ value indicating that all taxa are represented in the same relative concentrations. Both $H^{\prime}$ and $J^{\prime}$ were calculated according to the formulas found in Shannon \& Weaver (1949) and Krebs (1989). Statistical analyses of evenness and diversity indices were not performed because of low degrees of freedom; these indices are presented simply to show general patterns.
Based on Field et al.'s (1982) recommendation, we performed hierarchical cluster analyses in conjunction with non-metric multidimensional scaling (MDS) ordinations to identify potential taxa, station, cruise, depth and annual assemblages. For the taxa assemblage analyses, only those taxa $(n=30)$ occurring in $>5 \%$ of the hauls sampled were included, while all 71 identifiable taxa were included in the other assemblage analyses. Larval concentrations for each taxon were averaged for each haul $(\mathrm{n}=41)$, cruise $(\mathrm{n}=10)$ and depth stratum from all hauls ( $\mathrm{n}=188)$, which constituted the sampling units in the respective multivariate matrices. Sampling units with no taxa occurring were not included ( $\mathrm{n}=2$ for haul; $\mathrm{n}=66$ for depth stratum).

Taxon, station, cruise, depth and annual dendrograms were created using hierarchical, group-averaged clustering from Bray-Curtis ranked similarities on standardized, fourth root-transformed larval concentrations (Clarke \& Warwick 2001). The dendrograms were cut to produce ecologically interpretable clusters when and where they were apparent. To verify our interpretations of the dendrograms, we preformed non-metric MDS ordinations using the similarity matrices from the cluster analyses, with 20 random restarts each to minimize stress levels. A 2-dimensional ordination approach was adopted because stress levels were sufficiently low $(\leq 0.15)$ in all cases and were not appreciably reduced by the addition of a third dimension, and the results were adequately interpretable ecologically in 2-dimensional space (Clarke \& Warwick 2001).

A BIO-ENV, non-parametric, multivariate procedure was used to analyze the relationship between select environmental variables and larval community structure. The details of the BIO-ENV algorithm and its suitability for use in analyzing biological/environmental data interactions are described in Clarke \& Gorley (2001) and Clarke \& Warwick (2001). We performed 2 separate analyses, one using the station mean $\times$ taxa similarity matrix (41 stations $\times 71$ taxa), and the other using the depth-stratified sample $\times$ taxa matrix (196 samples $\times 71$ taxa). The station by taxa matrix was analyzed in association with 5 environmental variables: station location (distance from shore in $\mathrm{km}$, total watercolumn depth in $\mathrm{m})$, surface temperature $\left({ }^{\circ} \mathrm{C}\right)$, mean water-column temperature $\left({ }^{\circ} \mathrm{C}\right)$, surface salinity, and mean water-column salinity. The depth-stratified sample $\times$ taxa matrix was analyzed in association with 4 environmental variables: station location, mean depth (m), mean temperature and mean salinity of each depth-stratified sample. Both BIO-ENV analyses were performed using the Spearman rank-correlation method on the normalized Euclidean distance similarity matrices of the $\log _{\mathrm{e}}(\mathrm{N}+1)$-transformed, non-standardized environmental variables by haul or depthstratified sample (Clarke \& Gorley 2001). All diversity, 
evenness, cluster, MDS and BIO-ENV analyses were performed using PRIMER Version 5.2.9 statistical software (PRIMER-E 2001).

Pair-wise correlation analyses were also conducted to assess the relationship between concentrations of several prominent taxa (Engraulis mordax, Lyopsetta exilis, Sebastes spp., Stenobrachius leucopsarus and Tarletonbeania crenularis) and total larvae, and the environmental variables salinity and temperature. Mean larval concentrations, salinity and temperature per depth-stratified sample were used as variable measures. Prior to inclusion in the analyses, larval concentrations were $\log _{e}(N+0.1)$-transformed to normalize the data and homogenize residual variances. Pairs of variables were considered significantly correlated at the $\alpha=0.05$ level. All correlation analyses were performed using JMP IN Version 5.1 statistical software (SAS Institute 2005).

\section{RESULTS}

\section{Hydrography}

All sampling in both years occurred during periods of upwelling, with highest upwelling index values observed during July in each year (Fig. 2). Water temper-

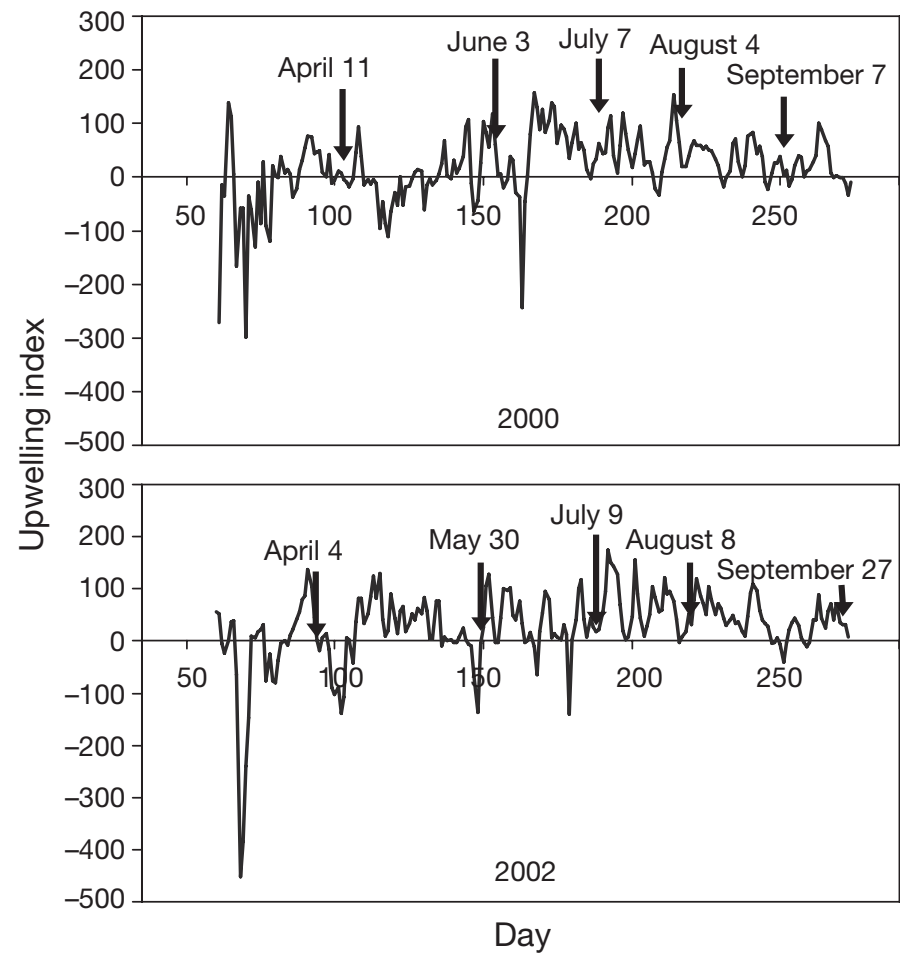

Fig. 2. Upwelling indices (UIs) off Oregon coast in 2000 and 2002. Sampling dates are indicated above arrows pointing to corresponding UI values. Upwelling indices obtained from the NOAA Pacific Fisheries Environmental Laboratory web site: http://www.pfeg.noaa.gov/ ature varied from 5.8 (150 m depth, Stn 46, April 2002) to $16.8^{\circ} \mathrm{C}$ (surface, Stn 84 , August 2000). Vertical thermal stratification existed across the shelf during each sampled month, increasing in intensity from April to September (Fig. 3). Surface water temperatures increased along an on-offshore gradient as a result of both upwelling and warm water from the Columbia River plume flowing in from the north. The influence of the Columbia River plume was evident in the pockets of high temperature, low salinity surface water observed offshore during most sampling months (Fig. 3). Salinity was inversely related to water temperature, and generally varied along similar upwelling-induced isoclines. Salinity varied from 29.3 (surface, Stn 46, April 2000) to 35.8 (150 m depth, Stn 46, April 2002) (station numbers represent $\mathrm{km}$ distance from shore).

\section{Larval collections, concentrations and distributions}

A total of 4944 fish larvae representing 72 taxa from 28 families were collected throughout the study (Table 2). Of these, 34 larvae (10 Engraulis mordax, 5 Sebastes spp., 1 Stenobrachius leucopsarus and 18 unidentified fish larvae) between 6 and $13 \mathrm{~mm}$ in length were found in the gastric cavities of ctenophores (Pleurobrachia spp.) and doliolid salps collected in the same samples. Four families accounted for $82.3 \%$ of the total standardized larval concentration: Myctophidae (29.4\%), Scorpaenidae (23.8\%), Pleuronectidae $(21.6 \%)$ and Engraulidae $(7.5 \%)$. Within these families, 5 taxa were the most dominant based on total mean concentration and frequency of occurrence from all hauls sampled: E. mordax, Lyopsetta exilis, Sebastes spp., Stenobrachius leucopsarus and Tarletonbeania crenularis. Osmerids were found at a high concentration (154.32 per $1000 \mathrm{~m}^{3}$ ) at a single coastal station (Stn 9) in April 2002, but were only found at much lower concentrations ( 2.24 to 3.87 per $1000 \mathrm{~m}^{3}$ ) on 3 other occasions (Stn 9, April 2000; Stn 28, April 2002; Stn 9, July 2002). Psettichthys melanostictus and Isopsetta isolepis were also found at relatively high mean concentrations but at low frequencies, whereas several other taxa occurred at relatively high frequencies but at lower mean concentrations: Liparis spp., Bathylagidae, Cottidae, Citharichthys spp. and Ammodytes hexapterus.

Total larval fish concentrations and distributions varied across annual, seasonal, coastal/offshore, and depth-stratified scales (Fig. 3). Mean concentrations of total larvae varied between 2000 and 2002 (Table 3). Larval Lyopsetta exilis concentrations varied 3-fold from 2000 to 2002, while larval Engraulis mordax varied >4-fold between 2000 and 2002. Sebastes spp., Stenobrachius leucopsarus and Tarletonbeania 

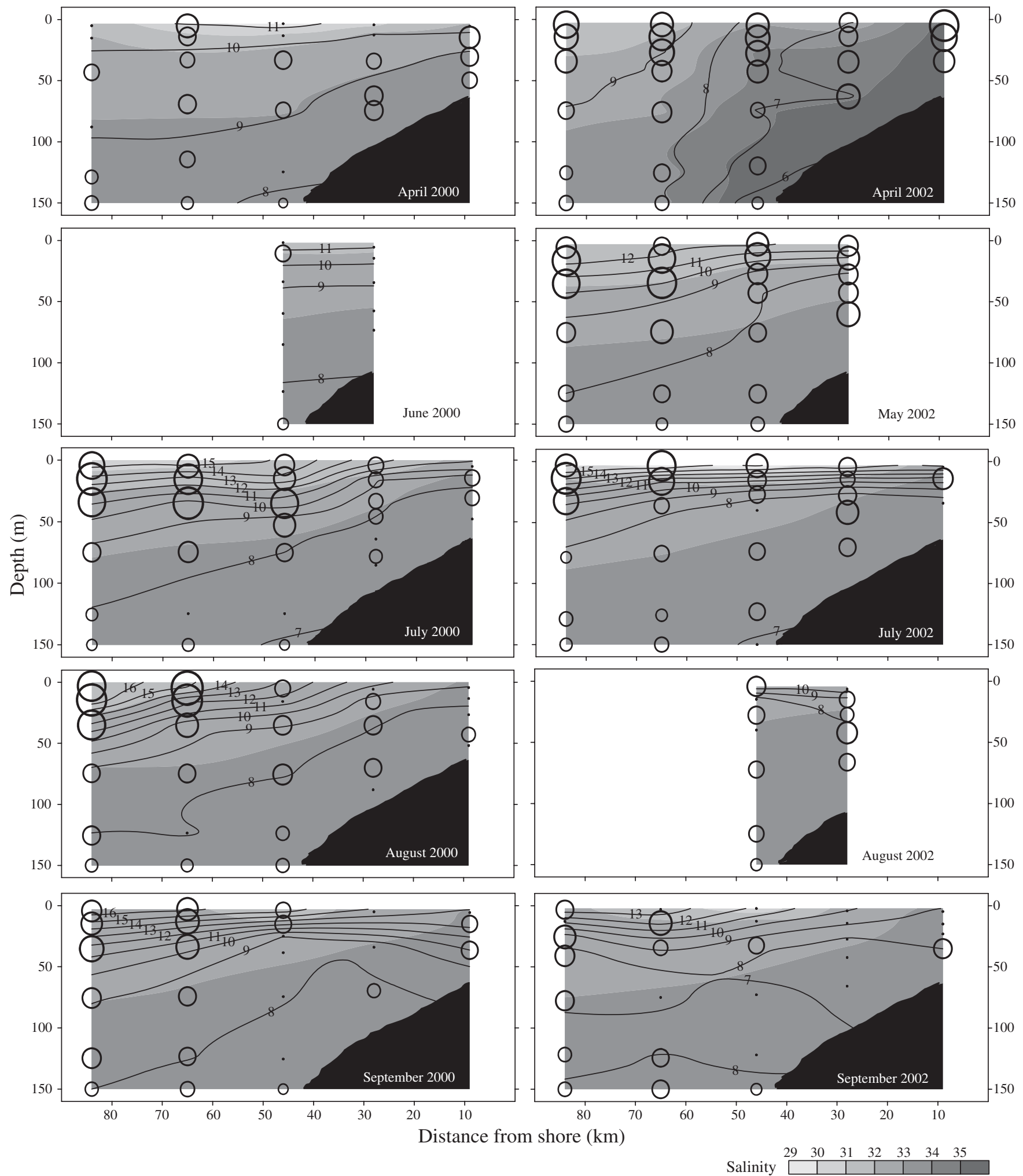

Fig. 3. Cross-section of sampled transect (= Newport Hydrographic line) depicting thermal (contour lines, $\mathrm{T}\left[{ }^{\circ} \mathrm{C}\right]$ ) and salinity (shading) structure, and relative concentrations of fish larvae (circles) throughout water column from April to September in 2000 and 2002; empty regions in June 2000, May 2002 and August 2002 represent station locations that were not sampled. Concentrations are $\log _{e}(\mathrm{~N}+0.1)$ relative (based on all collected samples), depth-stratified, concentrations (nos. per $1000 \mathrm{~m}^{3}$ ) of all fish larvae collected. Data for $150 \mathrm{~m}$ depth represent mean $\log _{\mathrm{e}}(\mathrm{N}+0.1)$ concentrations of all larvae collected at or below $150 \mathrm{~m}$ at each station 
Table 2. Species composition, frequency of occurrence, mean concentration and percent of total mean abundance for all larval fishes collected off Oregon coast from April through September in 2000 and 2002

\begin{tabular}{|c|c|c|c|c|c|}
\hline Taxa & & Common name & $\begin{array}{l}\text { Frequency } \\
\text { of } \\
\text { occurrence }\end{array}$ & $\begin{array}{c}\text { Mean con- } \\
\text { centration } \\
\text { (no. per } 1000 \mathrm{~m}^{3} \text { ) }\end{array}$ & $\begin{array}{c}\text { Total mean } \\
\text { abundance } \\
(\%)\end{array}$ \\
\hline Clupeiformes & Undetermined spp. & Herrings, anchovies & 0.02 & 0.09 & 0.15 \\
\hline Clupeidae & Sardinops sagax & Pacific sardine & 0.12 & 0.98 & 1.63 \\
\hline Engraulidae & Engraulis mordax & Northern anchovy & 0.26 & 4.51 & 7.47 \\
\hline Bathylagidae & $\begin{array}{l}\text { Pseudobathylagus milleri } \\
\text { Lipolagus ochotensis } \\
\text { Bathylagus pacificus } \\
\text { Undetermined spp. }\end{array}$ & $\begin{array}{l}\text { Stout blacksmelt } \\
\text { Eared blacksmelt } \\
\text { Pacific blacksmelt } \\
\text { Blacksmelts }\end{array}$ & $\begin{array}{l}0.14 \\
0.21 \\
0.02 \\
0.02\end{array}$ & $\begin{array}{l}0.11 \\
0.17 \\
0.01 \\
0.01\end{array}$ & $\begin{array}{l}0.18 \\
0.29 \\
0.02 \\
0.02\end{array}$ \\
\hline Osmeridae & Undetermined spp. & Smelts & 0.09 & 3.81 & 6.31 \\
\hline Phosichthyidae & Cyclothone signata & Showy bristlemouth & 0.02 & 0.01 & 0.01 \\
\hline Sternoptychidae & Undetermined spp. & Hatchetfishes & 0.05 & 0.02 & 0.04 \\
\hline Stomiidae & $\begin{array}{l}\text { Chauliodus macouni } \\
\text { Tactostoma macropus } \\
\text { Aristostomias scintillans }\end{array}$ & $\begin{array}{l}\text { Pacific viperfish } \\
\text { Longfin dragonfish } \\
\text { Shiny loosejaw }\end{array}$ & $\begin{array}{l}0.07 \\
0.02 \\
0.02\end{array}$ & $\begin{array}{l}0.18 \\
0.01 \\
0.01\end{array}$ & $\begin{array}{r}0.29 \\
<0.01 \\
0.01\end{array}$ \\
\hline Paralepididae & Lestidiops ringens & Slender barracudina & 0.02 & 0.02 & 0.03 \\
\hline Myctophidae & $\begin{array}{l}\text { Electrona rissoi } \\
\text { Protomyctophum crockeri } \\
\text { Protomyctophum thompsoni } \\
\text { Tarletonbeania crenularis } \\
\text { Nannobrachium regale } \\
\text { Stenobrachius leucopsarus } \\
\text { Diaphus theta }\end{array}$ & $\begin{array}{l}\text { Chubby flashlightfish } \\
\text { California flashlightfish } \\
\text { Northern flashlightfish } \\
\text { Blue lanternfish } \\
\text { Pinpoint lampfish } \\
\text { Northern lampfish } \\
\text { California headlightfish }\end{array}$ & $\begin{array}{l}0.02 \\
0.16 \\
0.26 \\
0.42 \\
0.26 \\
0.67 \\
0.26\end{array}$ & $\begin{array}{r}0.01 \\
0.13 \\
0.51 \\
2.39 \\
0.59 \\
13.64 \\
0.33\end{array}$ & $\begin{array}{r}0.02 \\
0.21 \\
0.85 \\
3.96 \\
0.98 \\
22.60 \\
0.54\end{array}$ \\
\hline Gadidae & Microgadus proximus & Pacific tomcod & 0.05 & 0.33 & 0.54 \\
\hline Bythitidae & Cataetyx rubrirostris & Rubynose brotula & 0.02 & 0.01 & 0.01 \\
\hline Trachipteridae & Trachipterus altivelis & King-of-the-salmon & 0.02 & 0.004 & $<0.01$ \\
\hline Melamphaidae & Undetermined spp. & Bigscales & 0.02 & 0.03 & 0.05 \\
\hline Scorpaenidae & $\begin{array}{l}\text { Sebastes spp. } \\
\text { Sebastolobus spp. }\end{array}$ & $\begin{array}{l}\text { Rockfishes } \\
\text { Thornyheads }\end{array}$ & $\begin{array}{l}0.63 \\
0.12\end{array}$ & $\begin{array}{c}14.21 \\
0.07\end{array}$ & $\begin{array}{r}23.53 \\
0.11\end{array}$ \\
\hline Anoplopomatidae & Anoplopoma fimbria & Sablefish & 0.02 & 0.01 & 0.01 \\
\hline Hexagrammidae & Ophiodon elongatus & Lingcod & 0.07 & 0.06 & 0.09 \\
\hline Cottidae & $\begin{array}{l}\text { Hemilepidotus hemilepidotus } \\
\text { Hemilepidotus spinosus } \\
\text { Hemilepidotus spp. } \\
\text { Ruscarius meanyi } \\
\text { Chitonotus pugetensis } \\
\text { Paricelinus hopliticus } \\
\text { Radulinus asprellus } \\
\text { Radulinus boleoides } \\
\text { Artedius fenestralis } \\
\text { Artedius harringtoni } \\
\text { Artedius corallinus or notospilotus } \\
\text { Leptocottus armatus }\end{array}$ & $\begin{array}{l}\text { Red Irish lord } \\
\text { Brown Irish lord } \\
\text { Irish lords } \\
\text { Puget Sound sculpin } \\
\text { Roughback sculpin } \\
\text { Thornback sculpin } \\
\text { Slim sculpin } \\
\text { Darter sculpin } \\
\text { Padded sculpin } \\
\text { Scalyhead sculpin } \\
\text { Coralline or bonyhead sculpin } \\
\text { Pacific staghorn sculpin }\end{array}$ & $\begin{array}{l}0.02 \\
0.05 \\
0.02 \\
0.05 \\
0.02 \\
0.02 \\
0.05 \\
0.02 \\
0.12 \\
0.19 \\
0.02 \\
0.02\end{array}$ & $\begin{array}{l}0.05 \\
0.01 \\
0.003 \\
0.16 \\
0.05 \\
0.01 \\
0.04 \\
0.04 \\
0.66 \\
1.03 \\
0.03 \\
0.03\end{array}$ & $\begin{array}{r}0.09 \\
0.02 \\
<0.01 \\
0.27 \\
0.07 \\
0.02 \\
0.07 \\
0.06 \\
1.09 \\
1.71 \\
0.05 \\
0.05\end{array}$ \\
\hline Psychrolutidae & Undetermined spp. & Fathead sculpins & 0.02 & 0.01 & 0.01 \\
\hline Agonidae & $\begin{array}{l}\text { Xeneretmus latifrons } \\
\text { Bathyagonus pentacanthus }\end{array}$ & $\begin{array}{l}\text { Blacktip poacher } \\
\text { Bigeye poacher }\end{array}$ & $\begin{array}{l}0.05 \\
0.02\end{array}$ & $\begin{array}{l}0.02 \\
0.01\end{array}$ & $\begin{array}{l}0.03 \\
0.02\end{array}$ \\
\hline Liparidae & $\begin{array}{l}\text { Liparis fucensis } \\
\text { Liparis mucosus } \\
\text { Liparis pulchellus } \\
\text { Liparis spp. }\end{array}$ & $\begin{array}{l}\text { Slipskin snailfish } \\
\text { Slimy snailfish } \\
\text { Showy snailfish } \\
\text { Snailfishes }\end{array}$ & $\begin{array}{l}0.47 \\
0.05 \\
0.02 \\
0.12\end{array}$ & $\begin{array}{l}0.72 \\
0.11 \\
0.05 \\
0.69\end{array}$ & $\begin{array}{l}1.19 \\
0.18 \\
0.08 \\
1.15\end{array}$ \\
\hline Bathymasteridae & Ronquilus jordani & Northern ronquil & 0.02 & 0.05 & 0.08 \\
\hline Stichaeidae & Plectobranchus evides & Bluebarred prickleback & 0.05 & 0.08 & 0.13 \\
\hline Pholidae & Pholis spp. & Gunnels & 0.02 & 0.04 & 0.06 \\
\hline Icosteidae & Icosteus aenigmaticus & Ragfish & 0.09 & 0.11 & 0.17 \\
\hline
\end{tabular}


Table 2 (continued)

\begin{tabular}{|c|c|c|c|c|c|}
\hline Taxa & & Common name & $\begin{array}{l}\text { Frequency } \\
\text { of } \\
\text { occurrence }\end{array}$ & $\begin{array}{c}\text { Mean con- } \\
\text { centration } \\
\left(\text { no. per } 1000 \mathrm{~m}^{3}\right)\end{array}$ & $\begin{array}{c}\text { Total mean } \\
\text { abundance } \\
(\%)\end{array}$ \\
\hline Ammodytidae & Ammodytes hexapterus & Pacific sand lance & 0.19 & 0.28 & 0.47 \\
\hline Centrolophidae & Icichthys lockingtoni & Medusafish & 0.09 & 0.03 & 0.05 \\
\hline Paralichthyidae & $\begin{array}{l}\text { Citharichthys sordidus } \\
\text { Citharichthys spp. }\end{array}$ & $\begin{array}{l}\text { Pacific sanddab } \\
\text { Sanddabs }\end{array}$ & $\begin{array}{l}0.21 \\
0.05\end{array}$ & $\begin{array}{l}0.36 \\
0.06\end{array}$ & $\begin{array}{l}0.59 \\
0.10\end{array}$ \\
\hline Pleuronectidae & $\begin{array}{l}\text { Embassichthys bathybius } \\
\text { Glyptocephalus zachirus } \\
\text { Hippoglossoides elassodon } \\
\text { Isopsetta isolepis } \\
\text { Lyopsetta exilis } \\
\text { Microstomus pacificus } \\
\text { Parophrys vetulus } \\
\text { Platichthys stellatus } \\
\text { Pleuronichthys decurrens } \\
\text { Psettichthys melanostictus }\end{array}$ & $\begin{array}{l}\text { Deepsea sole } \\
\text { Rex sole } \\
\text { Flathead sole } \\
\text { Butter sole } \\
\text { Slender sole } \\
\text { Dover sole } \\
\text { English sole } \\
\text { Starry flounder } \\
\text { Curlfin sole } \\
\text { Sand sole }\end{array}$ & $\begin{array}{l}0.02 \\
0.30 \\
0.05 \\
0.16 \\
0.56 \\
0.28 \\
0.19 \\
0.02 \\
0.02 \\
0.09\end{array}$ & $\begin{array}{l}0.01 \\
0.55 \\
0.05 \\
3.01 \\
4.38 \\
0.44 \\
0.75 \\
0.03 \\
0.003 \\
3.71\end{array}$ & $\begin{array}{r}0.01 \\
0.91 \\
0.08 \\
4.99 \\
7.26 \\
0.72 \\
1.24 \\
0.05 \\
<0.01 \\
6.15\end{array}$ \\
\hline Undetermined & & & 0.19 & 0.30 & 0.49 \\
\hline
\end{tabular}

crenularis larvae were found in relatively similar concentrations in 2000 and 2002 (Table 3).

Mean concentrations of Lyopsetta exilis, Sebastes spp., Stenobrachius leucopsarus, Tarletonbeania crenularis and total larvae were greater from April to May/June in 2002 than during the same period in 2000, but were less from July to September in 2002 than in 2000 (Table 3). The same seasonal shifts in peak concentrations were also apparent within each year, with greatest larval concentrations occurring during the summer of 2000 in contrast to the high spring concentrations in 2002. No Engraulis mordax larvae were collected from April to June in either year, or from August to September in 2002. Peak E. mordax concentrations shifted from August in 2000 (30.66 per $1000 \mathrm{~m}^{3}$ ) to July in 2002 (6.99 per $1000 \mathrm{~m}^{3}$ ). Total

Table 3. Survey-wide, annual, and monthly mean (SE) concentrations (no. per $1000 \mathrm{~m}^{3}$ ) of fish larvae collected off Oregon coast in 2000 and 2002. For between-month comparisons of each taxon within each year, different alphabetic superscripts indicate significant differences (ANOVA $\mathrm{p}<0.05$ ); for between-year comparisons of each taxon within each month and for annual totals, different numeric superscripts indicate significant differences (ANOVA $\mathrm{p}<0.05$ )

\begin{tabular}{|lccccrc}
\hline Taxon & April & May/June & July & August & September & Total \\
\hline 2000 & & & & & \\
Engraulis mordax & $0(0)$ & $0(0)$ & $0.48(0.23)$ & $30.66(18.82)$ & ${ }^{1} 0.65(0.34)$ & $7.22(4.80)$ \\
Lyopsetta exilis & $1.31(0.95)^{\mathrm{ab}}$ & ${ }^{2} 0(0)^{\mathrm{ab}}$ & $1.26(0.48)^{\mathrm{ab}}$ & $6.12(3.55)^{\mathrm{a}}$ & $0.58(0.58)^{\mathrm{b}}$ & $2.11(0.92)$ \\
Sebastes spp. & $20.71(0.71)$ & $20.58(0.58)$ & $55.72(28.59)$ & $10.78(5.66)$ & $2.86(1.85)$ & $15.98(7.73)$ \\
Stenobrachius leucopsarus & $5.85(3.58)$ & $20.26(0.26)$ & $21.40(12.75)$ & $24.71(18.77)$ & $4.72(3.05)$ & $12.91(5.24)$ \\
Tarletonbeania crenularis & $0(0)$ & $0(0)$ & $4.80(4.34)$ & $4.24(1.62)$ & $4.08(2.57)$ & $2.98(1.19)$ \\
Total larvae & $218.04(5.61)^{\mathrm{ab}}$ & ${ }^{2} 0.84(0.84)^{\mathrm{b}}$ & $87.72(35.77)^{\mathrm{a}}$ & $90.33(46.37)^{\mathrm{a}}$ & $20.67(8.63)^{\mathrm{ab}}$ & $49.34(14.73)$ \\
2002 & & & & & \\
Engraulis mordax & $0(0)^{\mathrm{b}}$ & $0(0)^{\mathrm{b}}$ & $6.99(6.31)^{\mathrm{a}}$ & $0(0)^{\mathrm{b}}$ & $20(0)^{\mathrm{b}}$ & $1.66(1.53)$ \\
Lyopsetta exilis & $13.00(10.63)^{\mathrm{ab}}$ & $115.59(9.52)^{\mathrm{a}}$ & $2.39(2.07)^{\mathrm{ab}}$ & $1.38(1.38)^{\mathrm{ab}}$ & $0(0)^{\mathrm{b}}$ & $6.77(3.21)$ \\
Sebastes spp. & $110.36(4.23)^{\mathrm{ab}}$ & $129.31(5.75)^{\mathrm{a}}$ & $11.74(4.55)^{\mathrm{ab}}$ & $10.59(7.53)^{\mathrm{ab}}$ & $2.12(1.32)^{\mathrm{b}}$ & $12.36(2.68)$ \\
Stenobrachius leucopsarus & $12.57(4.79)$ & $137.65(21.96)$ & $13.20(9.13)$ & $0.63(0.63)$ & $4.40(2.83)$ & $14.42(5.15)$ \\
Tarletonbeania crenularis & $0.25(0.17)$ & $5.34(3.11)$ & $2.41(1.42)$ & $0.28(0.28)$ & $0.39(0.29)$ & $1.77(0.75)$ \\
Total larvae & $1151.18(88.35)^{\mathrm{a}}$ & $1109.85(29.06)^{\mathrm{a}}$ & $44.61(13.68)^{\mathrm{ab}}$ & $15.76(6.19)^{\mathrm{ab}}$ & $12.23(4.56)^{\mathrm{b}}$ & $71.95(23.68)$ \\
2000 \& 2002 combined & & & & & \\
Engraulis mordax & $0(0)$ & $0(0)$ & $3.74(3.25)$ & $15.33(15.33)$ & $0.32(0.32)$ & $4.51(2.57)$ \\
Lyopsetta exilis & $7.15(5.85)$ & $7.80(7.80)$ & $1.82(0.56)$ & $3.75(2.37)$ & $0.29(0.29)$ & $4.38(1.66)$ \\
Sebastes spp. & $5.54(4.83)$ & $14.94(14.36)$ & $33.73(21.99)$ & $10.68(0.09)$ & $2.49(0.37)$ & $14.21(4.13)$ \\
Stenobrachius leucopsarus & $9.21(3.36)$ & $18.95(18.69)$ & $17.30(4.10)$ & $12.67(12.04)$ & $4.56(0.16)$ & $13.64(3.64)$ \\
Tarletonbeania crenularis & $0.13(0.13)$ & $2.67(2.67)$ & $3.61(1.19)$ & $2.26(1.98)$ & $2.24(1.84)$ & $2.39(0.71)$ \\
Total larvae & $84.61(66.57)$ & $55.35(54.51)$ & $66.17(21.55)$ & $53.04(37.28)$ & $16.45(4.22)$ & $60.38(13.74)$ \\
\hline
\end{tabular}


mean larval concentration was lowest in June 2000 (0.84 per $1000 \mathrm{~m}^{3}$ ) and highest in April 2002 (151.18 per $1000 \mathrm{~m}^{3}$ ). However, the high mean concentration found in April 2002 was influenced by the unusually high concentration of osmerid larvae found at a single coastal station during that month.

Mean larval concentrations were generally higher in offshore ( 46 to $84 \mathrm{~km}$ ) than coastal (9 to $28 \mathrm{~km}$ ) stations throughout the study (Table 4). However, this pattern was somewhat masked by the unusually high concentration of osmerid larvae found at coastal stations in April 2002. For the 2000 and combined years data, total larval concentrations were significantly higher at Stn 65 than at Stn 28 (ANOVA, p < 0.05) (Table 4). Larval concentrations of Sebastes spp. and Stenobrachius leucopsarus in both years increased along a coastaloffshore gradient to a maximum at Stn 65, whereas larval Tarletonbeania crenularis concentrations continued to increase out to Stn 84 . There were no significant coastal/offshore differences in larval Engraulis mordax and Lyopsetta exilis concentrations (ANOVA, $\mathrm{p}>0.05$ ), although $E$. mordax larvae were found in highest concentrations at Stn 65 throughout the study.

Almost $92 \%$ of the total larval fish abundance was distributed in the upper $100 \mathrm{~m}$ of the water column. Mean concentrations of Sebastes spp., Stenobrachius leucopsarus, Tarletonbeania crenularis and total larvae increased from the surface to $20 \mathrm{~m}$ depth, then declined steadily as depth increased (Table 5). In contrast, Lyopsetta exilis mean concentrations increased in most instances from the surface to maximum values at 50 to $100 \mathrm{~m}$ before declining. In the combined years data, mean concentrations of Engraulis mordax larvae were significantly higher in the upper $10 \mathrm{~m}$ of the water column than in all other depth strata (ANOVA, $p$ $<0.05$ ). In fact, no E. mordax larvae were found below $10 \mathrm{~m}$ in 2002 or below $50 \mathrm{~m}$ in 2000.

Mean standard length (SL) of Stenobrachius leucopsarus and Tarletonbeania crenularis larvae increased with increasing depth. For the combined years data, mean length of $S$. leucopsarus larvae collected below $100 \mathrm{~m}(15.5, \mathrm{SE}=1.1)$ was significantly higher than that of larvae collected from 50 to $100 \mathrm{~m}(10.8, \mathrm{SE}=0.4)$, and both were significantly higher than mean length of larvae collected from the 20 to $50 \mathrm{~m}(7.7, \mathrm{SE}=0.1), 10$ to $20 \mathrm{~m}(7.3, \mathrm{SE}=0.1)$, and 0 to $10 \mathrm{~m}(7.7, \mathrm{SE}=0.2)$ depth strata (ANOVA, $\mathrm{p}<0.05$ ). Similarly, mean lengths of $T$. crenularis larvae collected below $100 \mathrm{~m}$ $(14.6, \mathrm{SE}=1.0)$ and from 50 to $100 \mathrm{~m}(11.3, \mathrm{SE}=0.7)$ were significantly higher than lengths of larvae collected from the 20 to $50 \mathrm{~m}(7.4, \mathrm{SE}=0.3), 10$ to $20 \mathrm{~m}$ $(7.0, \mathrm{SE}=0.4)$, and 0 to $10 \mathrm{~m}(7.8, \mathrm{SE}=1.0)$ depth strata (ANOVA, $\mathrm{p}<0.05$ ). These trends were similar within stations, months and years. No consistent pattern of mean length differences between depths, stations, months or years was apparent for Engraulis mordax,

Table 4. Station ( $\mathrm{km}$ from shore) mean (SE) concentrations (no. per $1000 \mathrm{~m}^{3}$ ) of fish larvae collected off Oregon coast in 2000 and 2002. For between-station comparisons of each taxon within each year and survey-wide, different superscripts indicate significant differences (ANOVA $\mathrm{p}<0.05$ )

\begin{tabular}{|c|c|c|c|c|c|}
\hline Taxon & 9 & 28 & $\begin{array}{c}\text { Station No. } \\
46\end{array}$ & 65 & 84 \\
\hline \multicolumn{6}{|l|}{2000} \\
\hline Engraulis mordax & $0.28(0.28)$ & $0(0)$ & $0.17(0.11)$ & $20.92(20.02)$ & $18.32(18.00)$ \\
\hline Lyopsetta exilis & $1.43(0.68)$ & $5.06(3.87)$ & $1.42(0.98)$ & $0.81(0.40)$ & $1.24(0.73)$ \\
\hline Sebastes spp. & $0(0)^{\mathrm{b}}$ & $0.47(0.47)^{\mathrm{b}}$ & $21.26(19.74)^{\mathrm{ab}}$ & $44.24(33.67)^{\mathrm{a}}$ & $16.47(7.28)^{\mathrm{ab}}$ \\
\hline Stenobrachius leucopsarus & $0(0)^{\mathrm{d}}$ & $0.87(0.54)^{\mathrm{cd}}$ & $2.36(0.83)^{\mathrm{bc}}$ & $42.30(19.10)^{\mathrm{a}}$ & $24.66(14.00)^{\mathrm{ab}}$ \\
\hline Tarletonbeania crenularis & $0(0)$ & $0.48(0.48)$ & $0.63(0.63)$ & $4.87(2.31)$ & $10.13(4.69)$ \\
\hline Total larvae & $12.93(5.40)^{\mathrm{ab}}$ & $9.53(4.35)^{\mathrm{b}}$ & $27.98(20.39)^{\mathrm{ab}}$ & $120.16(49.31)^{\mathrm{a}}$ & $91.37(40.12)^{\mathrm{ab}}$ \\
\hline \multicolumn{6}{|l|}{2002} \\
\hline Engraulis mordax & $0(0)$ & $0.31(0.31)$ & $0.24(0.24)$ & $8.05(8.05)$ & $0(0)$ \\
\hline Lyopsetta exilis & $0(0)$ & $22.51(11.35)$ & $1.56(0.74)$ & $3.29(2.50)$ & $2.14(1.31)$ \\
\hline Sebastes spp. & $1.20(1.20)$ & $13.37(5.66)$ & $9.25(4.53)$ & $20.51(9.31)$ & $15.21(4.01)$ \\
\hline Stenobrachius leucopsarus & $0(0)^{b}$ & $3.46(2.41)^{\mathrm{b}}$ & $6.66(5.68)^{\mathrm{ab}}$ & $35.34(20.15)^{\mathrm{a}}$ & $27.70(11.27)^{\mathrm{a}}$ \\
\hline Tarletonbeania crenularis & $0(0)^{b}$ & $0(0)^{\mathrm{b}}$ & $0.50(0.27)^{\mathrm{ab}}$ & $4.20(2.38)^{\mathrm{ab}}$ & $4.47(2.60)^{\mathrm{a}}$ \\
\hline Total larvae & $177.78(161.27)$ & $57.03(23.13)$ & $23.19(9.18)$ & $81.42(34.69)$ & $62.73(21.06)$ \\
\hline \multicolumn{6}{|l|}{$2000 \& 2002$ combined } \\
\hline Engraulis mordax & $0.16(0.16)$ & $0.16(0.16)$ & $0.21(0.12)$ & $14.49(10.28)$ & $9.16(9.02)$ \\
\hline Lyopsetta exilis & $0.81(0.46)$ & $13.78(6.36)$ & $1.49(0.58)$ & $2.05(1.26)$ & $1.69(0.72)$ \\
\hline Sebastes spp. & $0.51(0.51)^{\mathrm{c}}$ & $6.92(3.43)^{\mathrm{bc}}$ & $15.26(9.76)^{\mathrm{abc}}$ & $32.37(16.78)^{\mathrm{a}}$ & $15.84(3.85)^{\mathrm{ab}}$ \\
\hline Stenobrachius leucopsarus & $0(0)^{\mathrm{c}}$ & $2.16(1.24)^{\mathrm{bc}}$ & $4.51(2.80)^{\mathrm{b}}$ & $38.82(12.92)^{\mathrm{a}}$ & $26.18(8.34)^{\mathrm{a}}$ \\
\hline Tarletonbeania crenularis & $0(0)^{\mathrm{c}}$ & $0.24(0.24)^{\mathrm{C}}$ & $0.57(0.33)^{\mathrm{bc}}$ & $4.54(1.54)^{\mathrm{ab}}$ & $7.30(2.70)^{\mathrm{a}}$ \\
\hline Total larvae & $83.58(69.52)^{\mathrm{ab}}$ & $33.28(13.63)^{b}$ & $25.58(10.57)^{\mathrm{ab}}$ & $100.79(28.85)^{\mathrm{a}}$ & $77.05(21.66)^{\mathrm{ab}}$ \\
\hline
\end{tabular}


Table 5. Depth-stratified mean (SE) concentrations (no. per $1000 \mathrm{~m}^{3}$ ) of fish larvae collected off Oregon coast in 2000 and 2002. For between depth stratum comparisons of each taxon within each year and survey-wide, different superscripts indicate significant differences (ANOVA $p<0.05$ )

\begin{tabular}{|c|c|c|c|c|c|}
\hline Taxon & $0-10 \mathrm{~m}$ & $10-20 \mathrm{~m}$ & $20-50 \mathrm{~m}$ & $50-100 \mathrm{~m}$ & $>100 \mathrm{~m}$ \\
\hline \multicolumn{6}{|l|}{2000} \\
\hline Engraulis mordax & $147.53(111.87)^{\mathrm{a}}$ & $55.81(54.16)^{\mathrm{ab}}$ & $10.94(10.72)^{b}$ & $0(0)^{\mathrm{b}}$ & $0(0)^{b}$ \\
\hline Lyopsetta exilis & $0.43(0.43)^{\mathrm{b}}$ & $0(0)^{\mathrm{b}}$ & $4.69(1.94)^{a}$ & $3.96(1.27)^{\mathrm{a}}$ & $0(0)^{b}$ \\
\hline Sebastes spp. & $56.69(31.40)^{\mathrm{a}}$ & $84.88(50.82)^{\mathrm{a}}$ & $116.33(70.92)^{\mathrm{a}}$ & $5.33(3.41)^{\mathrm{ab}}$ & $0.17(0.14)^{\mathrm{b}}$ \\
\hline Stenobrachius leucopsarus & $44.93(40.14)$ & $161.34(112.61)$ & $57.15(25.67)$ & $3.81(1.59)$ & $3.14(1.81)$ \\
\hline Tarletonbeania crenularis & $5.38(4.89)$ & $41.07(29.44)$ & $11.15(4.81)$ & $2.14(1.12)$ & $0.93(0.57)$ \\
\hline Total larvae & $287.02(193.43)^{\mathrm{ab}}$ & $414.06(181.71)^{\mathrm{a}}$ & $211.73(91.36)^{\mathrm{a}}$ & $21.35(5.28)^{\mathrm{ab}}$ & $8.00(2.64)^{b}$ \\
\hline \multicolumn{6}{|l|}{2002} \\
\hline Engraulis mordax & $48.05(45.75)^{\mathrm{a}}$ & $0(0)^{\mathrm{b}}$ & $0(0)^{\mathrm{b}}$ & $0(0)^{\mathrm{b}}$ & $0(0)^{\mathrm{b}}$ \\
\hline Lyopsetta exilis & $1.01(0.70)^{\mathrm{bc}}$ & $7.28(3.45)^{\mathrm{ab}}$ & $8.58(2.29)^{\mathrm{a}}$ & $16.42(7.55)^{\mathrm{a}}$ & $0.14(0.08)^{\mathrm{c}}$ \\
\hline Sebastes spp. & $33.21(10.48)^{\mathrm{ab}}$ & $79.84(31.26)^{\mathrm{a}}$ & $48.01(16.29)^{\mathrm{a}}$ & $6.77(2.48)^{\mathrm{ab}}$ & $2.26(0.65)^{\mathrm{b}}$ \\
\hline Stenobrachius leucopsarus & $17.90(9.89)^{\mathrm{ab}}$ & $137.51(62.51)^{\mathrm{a}}$ & $60.01(30.72)^{\mathrm{ab}}$ & $5.99(3.40)^{\mathrm{ab}}$ & $2.41(0.94)^{b}$ \\
\hline Tarletonbeania crenularis & $2.39(1.65)$ & $9.31(6.97)$ & $9.38(4.75)$ & $1.28(0.89)$ & $0.26(0.15)$ \\
\hline Total larvae & $208.90(95.52)^{\mathrm{ab}}$ & $317.12(98.72)^{\mathrm{a}}$ & $143.82(53.60)^{\mathrm{a}}$ & $45.51(15.22)^{\mathrm{ab}}$ & $8.58(1.27)^{\mathrm{b}}$ \\
\hline \multicolumn{6}{|l|}{$2000 \& 2002$ combined } \\
\hline Engraulis mordax & $98.95(61.21)^{\mathrm{a}}$ & $27.90(27.10)^{b}$ & $4.90(4.81)^{\mathrm{b}}$ & $0(0)^{b}$ & $0(0)^{\mathrm{b}}$ \\
\hline Lyopsetta exilis & $0.71(0.40)^{\mathrm{c}}$ & $3.64(1.80)^{\mathrm{bc}}$ & $6.84(1.54)^{\mathrm{ab}}$ & $8.95(3.20)^{\mathrm{a}}$ & $0.08(0.04)^{\mathrm{c}}$ \\
\hline Sebastes spp. & $45.22(16.76)^{\mathrm{a}}$ & $82.36(29.47)^{\mathrm{a}}$ & $78.63(33.00)^{a}$ & $5.91(2.25)^{\mathrm{ab}}$ & $1.27(0.36)^{b}$ \\
\hline Stenobrachius leucopsarus & $31.73(20.96)^{\mathrm{ab}}$ & $149.42(63.63)^{\mathrm{a}}$ & $58.72(20.32)^{a}$ & $4.68(1.65)^{\mathrm{ab}}$ & $2.76(0.98)^{b}$ \\
\hline Tarletonbeania crenularis & $3.92(2.61)^{\mathrm{ab}}$ & $25.19(15.15)^{\mathrm{ab}}$ & $10.17(3.37)^{\mathrm{a}}$ & $1.79(0.75)^{\mathrm{ab}}$ & $0.58(0.28)^{\mathrm{b}}$ \\
\hline Total larvae & $248.87(108.30)^{\mathrm{ab}}$ & $365.59(102.41)^{\mathrm{a}}$ & $174.26(50.23)^{\mathrm{a}}$ & $31.01(6.99)^{\mathrm{ab}}$ & $8.30(1.41)^{b}$ \\
\hline
\end{tabular}

Lyopsetta exilis or Sebastes spp. larvae. In addition, there were no significant differences in distributions, concentrations, or mean lengths of dominant and total larval taxa across annual, seasonal, coastal/offshore or depth-stratified scales that could be attributed to day versus night collections (ANOVA, p > 0.05).

\section{Diversity and evenness}

Diversity and evenness varied across annual, seasonal, coastal/offshore and depth-stratified scales (Fig. 4). Diversity and evenness were generally highest in April and September, although highest evenness and lowest diversity were observed in June 2000. Lowest diversity and evenness occurred at a station $46 \mathrm{~km}$ offshore, but were fairly uniform across all other stations. Diversity and evenness generally decreased from the surface to $50 \mathrm{~m}$, then increased with increasing depth. However, lowest diversity occurred in the 0 to $10 \mathrm{~m}$ depth stratum in 2000 .

\section{Assemblages}

Several species, station, and seasonal assemblages were identified based on cluster analyses and multidimensional scaling (MDS) (Figs. 5 \& 6), although no interannual or depth-stratified assemblages were apparent. Species separated out into 5 assemblages: coastal, offshore, Columbia River plume, and 2 clusters based on single species, Pseudobathylagus milleri and Chauliodus macouni (Figs. 5A \& 6A). The P. milleri, C. macouni and plume clusters could further be grouped together with the offshore assemblage to form 2 distinct assemblages: coastal and offshore. This dual assemblage interpretation was reinforced by the occurrence of 2 station assemblages: coastal (9 to 28 $\mathrm{km}$ from shore) and offshore (46 to $84 \mathrm{~km}$ from shore) (Figs. 5B \& 6B). Cluster analyses and MDS also suggested the presence of 3 seasonal assemblages based on sampling cruises: spring, summer, and fall (Figs. 5C \& 6C). The May 2002 and September 2000 cruises fell within the summer assemblage due to the late timing of the May 2002 cruise (May 30) and the early timing of the September 2000 cruise (September 7).

\section{Environmental relationships}

BIO-ENV and correlation analyses revealed significant relationships between several environmental factors and mean concentrations of larval fishes. A station-based BIO-ENV multivariate analysis including the environmental variables station location (distance from shore in $\mathrm{km}$, total water-column depth in $\mathrm{m}$ ), sur- 

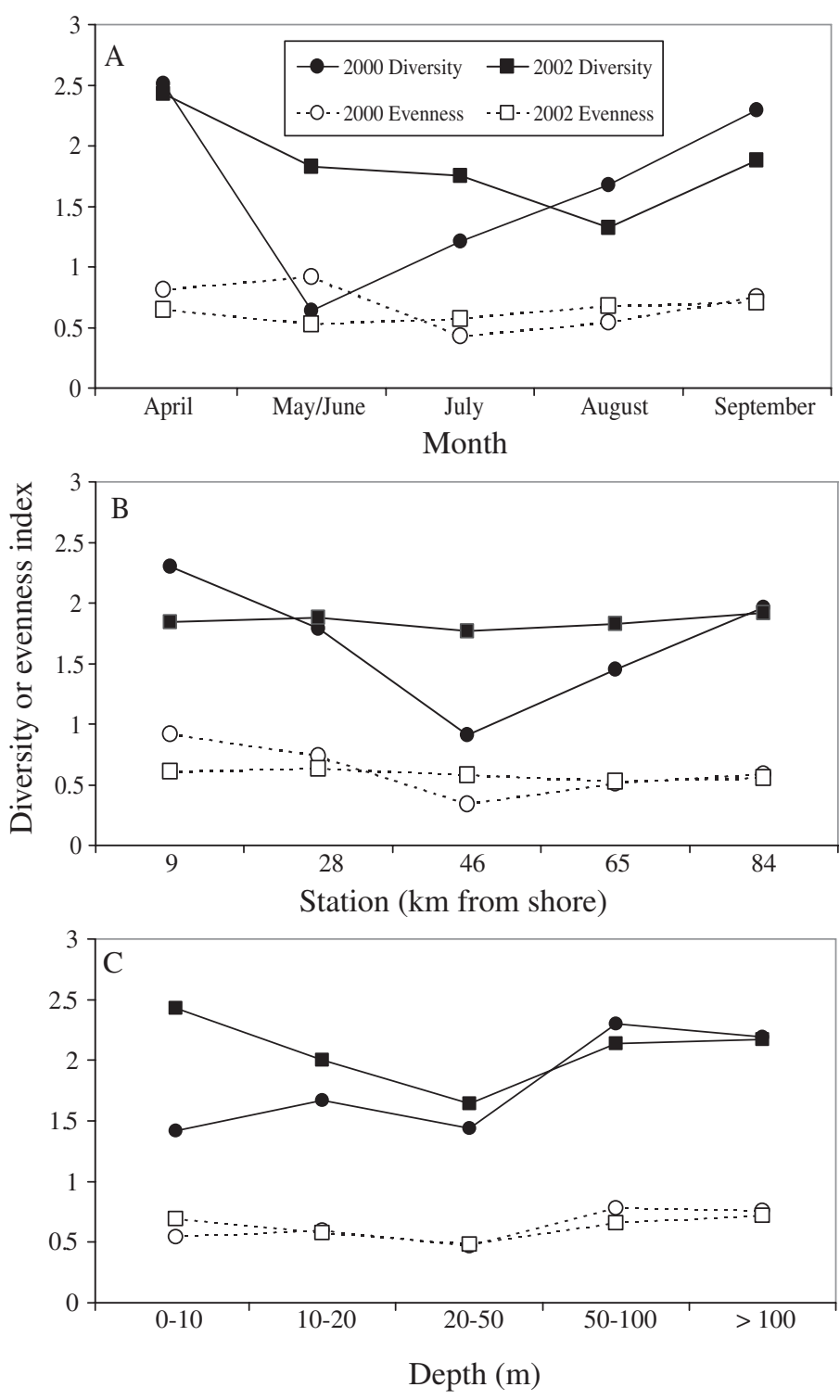

Fig. 4. Taxon diversity and evenness indices for larval fishes collected off Oregon coast in 2000 and 2002 by month, station ( $\mathrm{km}$ from shore) and depth $(\mathrm{m})$. Species diversity measured by Shannon-Wiener diversity index $\left(H^{\prime}\right)$, whereby greater $H^{\prime}=$ greater diversity. Species evenness measured by Pielou's evenness index $\left(J^{\prime}\right) ; J^{\prime}$ has maximum value of 1 , indicating that all taxa are present in same relative concentrations

face temperature $\left({ }^{\circ} \mathrm{C}\right)$, mean water-column temperature $\left({ }^{\circ} \mathrm{C}\right)$, surface salinity and mean water-column salinity showed that station location alone explained $59 \%$ of the variability in mean larval fish concentrations. No multiple-factor combination explained more variability in the larval concentration data. A depth-stratified BIO-ENV analysis including the environmental variables station location, mean depth (m), mean temperature, and mean salinity of each depth-stratified sample also showed that station location was the dominant environmental factor, explaining $28 \%$ of the variability in mean larval fish concentrations. The only multiple-factor combination that explained more variability in the depth-stratified larval concentration data was station location and mean depth (29\%). Pair-wise correlation analyses revealed that mean concentrations of Engraulis mordax, Sebastes spp., Stenobrachius leucopsarus, Tarletonbeania crenularis and total larvae were significantly positively correlated with temperature and negatively correlated with salinity, and that temperature and salinity were negatively correlated with each other ( $p<0.0001)$ (Table 6). However, there were no significant correlations between either temperature or salinity and mean concentration of Lyopsetta exilis larvae.

\section{DISCUSSION}

The overall species composition and distribution of larval fishes identified in this study were characteristic of previous studies conducted over the last 30 yr (Richardson 1973, Richardson \& Pearcy 1977, Mundy 1984, Boehlert et al. 1985, Brodeur et al. 1985, Doyle 1992), although we did observe some notable differences. One species, Sardinops sagax, was not collected off Oregon during all the previous $\mathrm{NH}$ line sampling and has only been known to spawn in this region since the 1990s (Emmett et al. 2005). Through recent advances in our knowledge of larval fish identification, many taxa that had been identified only to family or type (e.g. Cottidae and Cyclopteridae) in early studies are now identifiable to species (Matarese et al. 2003). Other groups (Osmeridae and Sebastes) remain problematic and are classified only to higher taxonomic levels (see Richardson \& Pearcy 1977 for more complete discussion). Thus, our estimates of species diversity are likely to be low, and distributional analyses involving these taxa may be somewhat clouded since they could represent multiple species.

Table 6. Correlation coefficients for depth-stratified sample means $(n=281)$ of 8 variables sampled off Oregon coast in 2000 and 2002: water temperature $\left({ }^{\circ} \mathrm{C}\right)$, salinity and $\log _{\mathrm{e}}-$ transformed concentrations (no. per $1000 \mathrm{~m}^{3}$ ) of Engraulis mordax, Lyopsetta exilis, Sebastes spp., Stenobrachius leucopsarus, Tarletonbeania crenularis, and total larvae. ${ }^{*} \mathrm{p}<0.0001$

\begin{tabular}{|lcc|}
\hline & Temperature & Salinity \\
\hline Salinity & $-0.80^{*}$ & - \\
Engraulis mordax & $0.56^{*}$ & $-0.34^{*}$ \\
Lyopsetta exilis & -0.04 & -0.06 \\
Sebastes spp. & $0.38^{*}$ & $-0.40^{*}$ \\
Stenobrachius leucopsarus & $0.25^{*}$ & $-0.26^{*}$ \\
Tarletonbeania crenularis & $0.26^{*}$ & $-0.23^{*}$ \\
Total larvae & $0.31^{*}$ & $-0.25^{*}$ \\
\hline
\end{tabular}


A

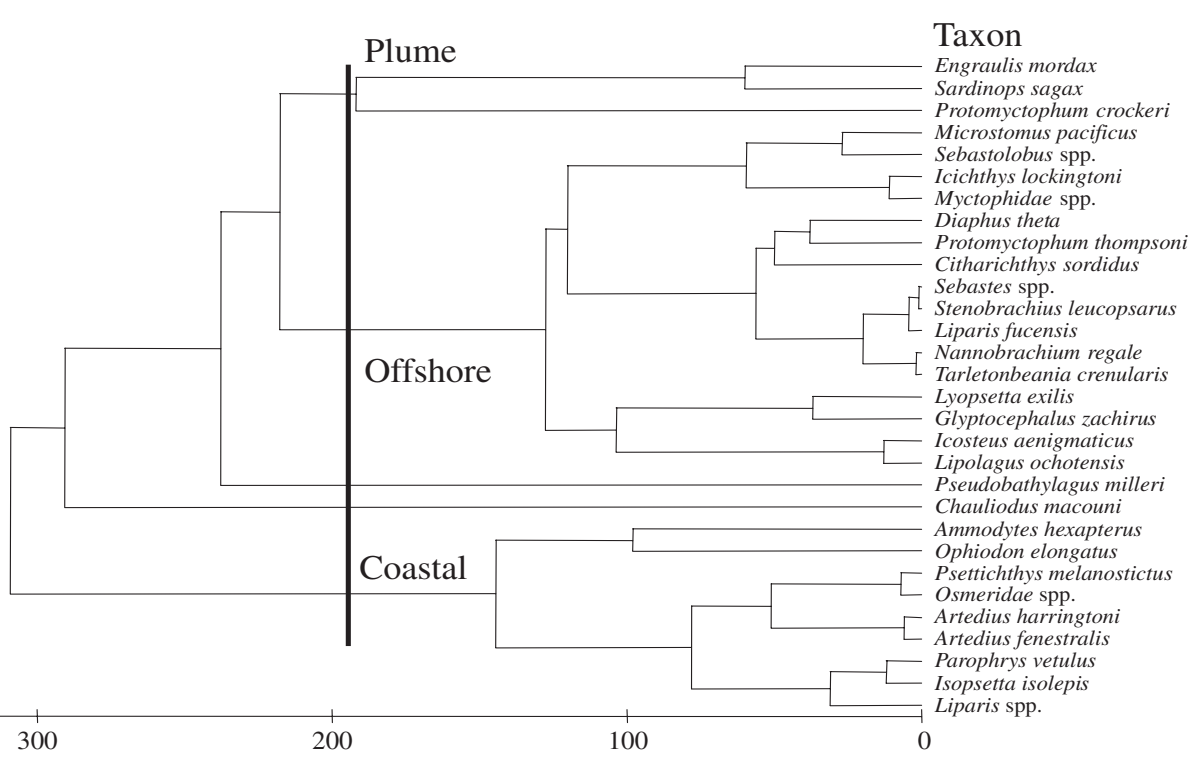

B

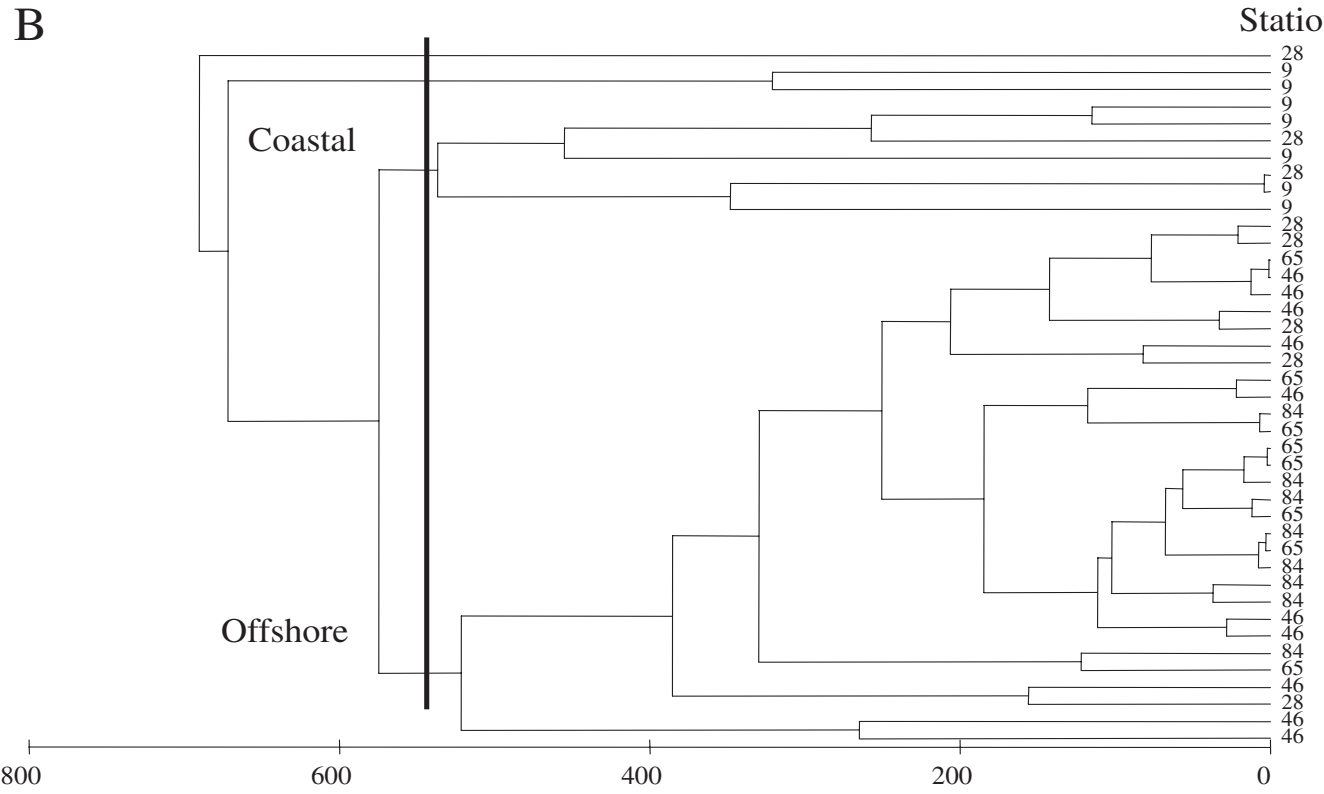

$\mathrm{C}$

Cruise

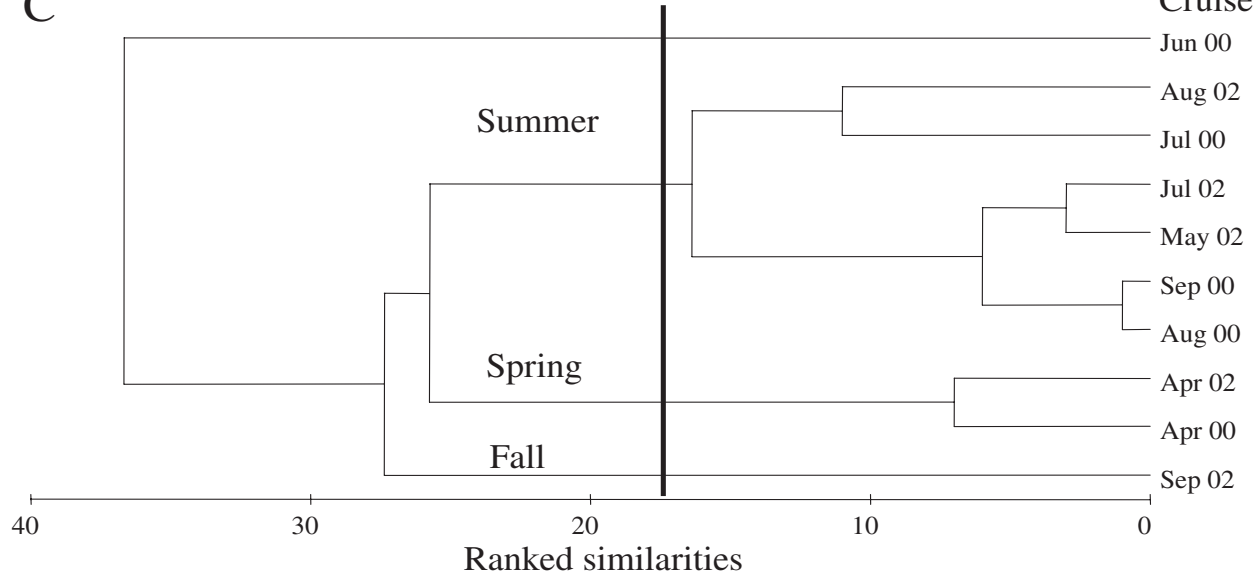

Fig. 5. Taxon, station and cruise dendrograms resulting from cluster analyses performed on larval fishes collected off Oregon coast in 2000 and 2002. Ecologically interpretable cluster identifications are indicated on each figure 


\section{Seasonal and interannual abundance patterns}

Seasonal changes in occurrence and abundance are expected in temperate systems such as the northern California Current, where spawning times of different species vary depending on their reproductive strategies in relation to varying oceanographic conditions (Parrish et al. 1981). Generally, most species have fairly predictable periods of occurrence in the plankton, although some taxa (including several pleuronectids) have prolonged spawning seasons and extended larval phases, and can occur in plankton samples throughout the year (Pearcy et al. 1977, Ureña 1989). Despite the similar sampling times in the 2 years of this study, there were some substantial interannual differences in larval abundances that could be related to changes in the timing or location of spawning, or alternatively to changes in oceanographic conditions that affected transport and survival of larvae. The relative importance of these factors is not likely to be interpretable based on sampling from a single transect. There is a substantial evolution of hydrographic and productivity regimes during the course of the summer off Newport, but the flow is predominantly to the south during most of the upwelling period (Barth et al. 2005). Chlorophyll a concentrations were substantially higher off Newport in 2002 than in 2000 (Wheeler et al. 2003, Suchman \& Brodeur 2005) due to anomalous southward transport of high-nutrient cold water from the subarctic region in 2002 (Kosro 2003). The dense phytoplankton blooms sunk to the bottom, resulting in hypoxic conditions in the lower 15 to $30 \mathrm{~m}$ of the water column over much of this region, beginning in August 2002 (Grantham et al. 2004). It is uncertain what effect this hypoxia had on fish larvae, but we noted a substantial drop in concentrations after July 2002, which was dissimilar to the seasonal pattern that occurred during the hydrographically more normal summer of 2000 . However, the earlier occurrence of peak larval concentrations in 2002 (April and May) relative to 2000 (July and August) coincided more closely with the data of Richardson \& Pearcy (1977), who found that $24 \%$ of all larvae were taken in February and March, $68 \%$ in May to July, and only $8 \%$ in August to December along the NH line in 1971 and 1972. This led us to believe that 2002 was a more 'normal' year than 2000 with respect to seasonal larval fish distributions.

\section{Zonal distribution patterns}

Station location (an index of water column depth and distance from shore) was the most important factor in structuring larval fish assemblages off the central
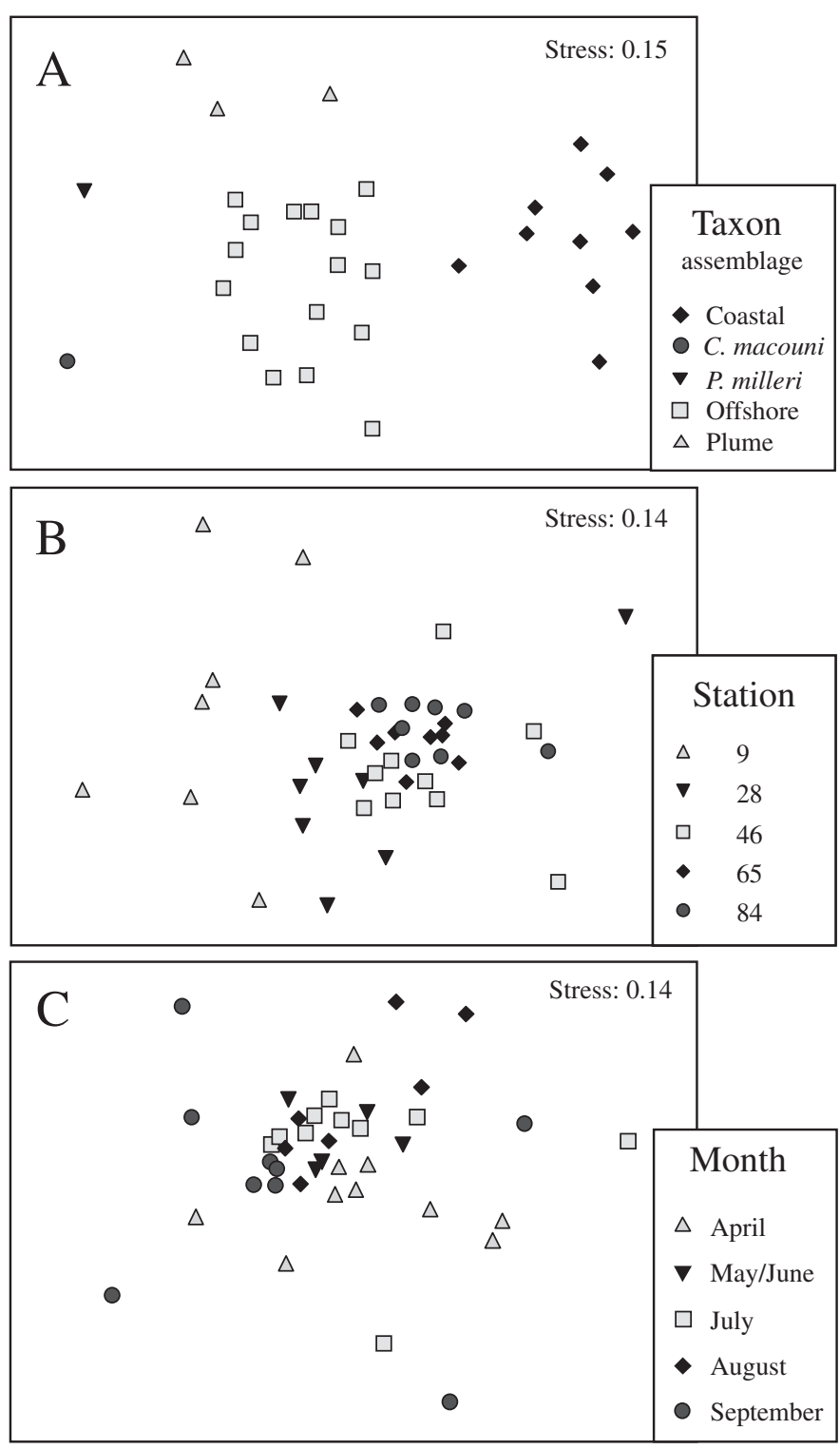

Fig. 6. Taxon, station and cruise plots resulting from multidimensional scaling analyses performed on larval fishes collected off Oregon coast in 2000 and 2002. Ecologically interpretable cluster identifications are indicated on each graph. C. macouni $=$ Chauliodus macouni; $P$. milleri $=$ Pseudobathylagus milleri

Oregon coast. The cross-shelf distribution patterns of the taxa we observed may be largely dictated by the location of adult spawning. Larvae of several dominant species in the region had extended horizontal distributions, which indicated that adults spawned mainly near the coast and that eggs and larvae were carried offshore by surface Ekman transport associated with coastal upwelling. This is supported by our findings that the majority of larval taxa collected exhibited increased mean lengths in a coastal-offshore direction, although sample sizes were often too small to deter- 
mine statistical significance. However, although there is some offshore transport at the surface during the upwelling season, the transport of satellite-tracked drifters released on the $\mathrm{NH}$ line and the flow at $25 \mathrm{~m}$ determined with a shipboard acoustic Doppler currentprofiler were predominantly to the south during the summer months (Barth et al. 2005). Osmerids, sculpins, greenlings and some flatfishes are predominantly coastal spawners, and their larvae were representative of the coastal assemblage. Ureña (1989) found that coastal pleuronectids (such as Isopsetta isolepis, Parophrys vetulus and Psettichthys melanostictus, which we collected primarily at the stations nearest shore) used a combination of retention mechanisms. These included spawning close to shore in winter during downwelling periods, and avoidance of areas of intense upwelling and the near-surface zone to maintain their progeny in the coastal zone. In contrast, many spring-spawning rockfishes and some pleuronectids (Lyopsetta exilis and Glyptocephalus zachirus) were collected mainly at the transitional outer-shelf stations. Northern anchovies were predominantly collected offshore and were most probably associated with the Columbia River plume, which intersected the $\mathrm{NH}$ line far offshore during our sampling periods. Other larvae occurring offshore such as sardines, bathylagids and myctophids are likely to be spawned well off the shelf break, and the conditions during this study were generally not amenable to coastal transport.

\section{Depth distribution patterns}

Our study was not explicitly designed to determine diel distribution patterns of ichthyoplankton in this region. To accomplish this, we would have had to sample the same location at multiple times during the day to disentangle the spatial and temporal variations in their distribution. Diel vertical migrations of these dominant species will be the subject of a subsequent study dealing with another location where repeated sampling did occur. However, we are able to draw some conclusions about vertical distribution based on our present results that may benefit future sampling efforts. For example, Engraulis mordax and Sardinops sagax larvae were found almost entirely in the upper $20 \mathrm{~m}$ of the water column. Other species, including rockfishes and myctophids, were more abundant at intermediate depths but still above the pycnocline, as found in previous studies (Richardson 1973, Boehlert et al. 1985, Sakuma et al. 1999). The nets that sampled below $100 \mathrm{~m}$ caught relatively few larvae overall, which led us to conclude that sampling the upper 100 $\mathrm{m}$ of the water column is sufficient to characterize the larval fish community during the upwelling season.

\section{Community patterns}

The assemblages we observed in this study were not substantially different from those documented in the 1970 s and 1980s with respect to their cross-shelf distributions as determined by assemblage analysis (Richardson \& Pearcy 1977, Richardson et al. 1980, Doyle et al. 2002). According to Richardson \& Pearcy (1977), ichthyoplankton have been classified as coastal ( $>80 \%$ of their larvae occur between 2 and $28 \mathrm{~km}$ ) or offshore (>80\% caught between 37 and $111 \mathrm{~km}$ ), although Richardson et al. (1980) also identified a transitional assemblage along the $200 \mathrm{~m}$ isobath (shelf break) in March and April. Doyle et al. (2002) analyzed data from a much broader area of the northern California Current for 2 yr (spring of 1980 and of 1981) and also found 3 assemblages including coastal/shelf, slope and oceanic groups. There was only a moderate amount of fidelity within these groups, as some species such as Stenobrachius leucopsarus were important to all 3 assemblages. Moreover, relaxation of upwelling during some years and anomalous coastal flow patterns, such as those that occur during El Niño conditions, can push normally offshore taxa such as northern anchovy, bathylagids and myctophids into coastal regions (Brodeur et al. 1985, Doyle 1995). This is consistent with the observed distribution patterns for zooplankton (Keister \& Peterson 2003, Morgan et al. 2003, Gómez-Gutiérrez et al. 2005) and nekton (Brodeur et al. 2004, 2005) in the northern California Current. Strong cross-shelf zonal patterns are commonly observed in other upwelling and non-upwelling areas as well (Olivar 1990, Sebatés 1990, Cowen et al. 1993, Marancik et al. 2005). We also identified a plume assemblage consisting of Engraulis mordax and Sardinops sagax larvae associated with warmer, less saline, offshore surface water from the Columbia River (Richardson 1973). In addition, this assemblage was only apparent during the summer months in both the present and in Richardson's (1973) study.

\section{Diversity patterns}

Differential seasonal spawning periods and the presence of coastal and offshore assemblages largely influenced species diversity. The high diversity observed during April and September in both survey years reflects the peak spring and fall spawning seasons of the majority of coastal Oregon fish taxa (Matarese et al. 2003). The abnormally low diversity observed in June 2000, together with the highest evenness and lowest larval concentrations observed in any single month during the study, may have resulted from unfavorable environmental conditions for spawning and/or 
larval survival in the days before the samples were collected. Alternatively, the low diversities observed in June 2000 and August 2002 may be the result of low sampling effort during these cruises (Table 1). The pattern of higher larval diversity at the extreme coastal and offshore stations (more apparent in 2000 than in 2002) than at the intermediate station $46 \mathrm{~km}$ off the coast may be related to the presence of 2 distinct assemblages on either side of the shelf break, as noted earlier. This seems to be counterintuitive, as we anticipated that there would be higher diversity in the transitional region where the distributions of both assemblages would be expected to overlap. However, as Gómez-Gutiérrez et al. (2005) found for euphausiids on the same transect, there do not appear to be any ichthyoplankton taxa that are indicative of this transitional zone or have adapted to living there.

\section{Ecological implications}

Our observation of developed larvae within the gastric cavity of ctenophores and salps is noteworthy, as there have been few documented examples of predation on any fish larvae in the northern California Current. Although it is possible that some of these larvae were consumed in the codend of the plankton net, the advanced stage of digestion coupled with the low net/handling time (<30 min) would argue against this artificial predation mechanism. Gelatinous zooplankton are acknowledged to be important predators of fish larvae in many marine ecosystems (Purcell \& Arai 2001), and the consumption of even a few fish larvae by these extremely abundant predators would suggest that this is an area worthy of further study.

Although our discrete depth sampling, consisting of relatively low volumes of water sampled with each net, afforded us a more detailed picture of the spatial structure of ichthyoplankton off the central Oregon coast than has heretofore been available, we realize that our nets still integrate fairly large volumes. We also sampled at predetermined stations, and if larval concentrations are patchily distributed with respect to hydrographic features such as fronts (e.g. Bjorkstedt et al. 2002), our concentration estimates may be biased on the low side compared to actual in situ concentrations. This uncertainty in fine-scale distributions limits our ability to examine competition even among larvae of different species caught in the same tow.

Acknowledgements. We thank the captains and crews of all research vessels participating in this study for their cooperation and assistance in the sampling. Collection of all MOCNESS tows discussed in this paper was made possible through two grants (NA 67RJ10151 and NA 86OP0589) to Bill
Peterson from the US GLOBEC program. We are indebted to the Peterson Zooplankton Group, in particular Julie Keister, Mitch Vance, Leah Feinberg, Tracy Shaw, Jesse Lamb, Anders Røestad and Daryl Swenson, for their efforts in collecting the at-sea data and for archiving the samples. We thank Maria Parnel for assistance with identification of larvae, and Edmundo Casillas, Miriam Doyle, Bill Peterson and Julie Keister for critical reviews of the manuscript. Funding was provided by the Northeast Pacific GLOBEC Program, and this is contribution number 287 of the US GLOBEC Program.

\section{LITERATURE CITED}

Barth JA, Pierce SD, Cowles TJ (2005) Mesoscale structure and its seasonal evolution in the northern California Current System. Deep-Sea Res II 52:5-28

Batchelder HP, Barth JA, Kosro PM, Strub PT and 11 others (2002) The GLOBEC Northeast Pacific California Current system program. Oceanogr 15:36-47

Bjorkstedt EP, Rosenfield LK, Grantham BA, Shkedy Y, Roughgarden J (2002) Distributions of larval rockfishes Sebastes spp. across nearshore fronts in a coastal upwelling region. Mar Ecol Prog Ser 242:215-228

Boehlert GW, Gadomski GM, Mundy BC (1985) Vertical distribution of ichthyoplankton off the Oregon Coast in spring and summer months. Fish Bull US 83:611-621

Brodeur RD, Gadomski DM, Pearcy WG, Batchelder HP, Miller CB (1985) Abundance and distribution of ichthyoplankton in the upwelling zone off Oregon during anomalous El Niño conditions. Estuar Coast Shelf Sci 21: 365-378

Brodeur RD, Fisher JP, Teel D, Emmett RL, Casillas E, Miller TW (2004) Distribution, growth, condition, origin and environmental and species associations of juvenile salmonids in the Northern California Current. Fish Bull US 102:25-46

Brodeur RD, Fisher JP, Morgan CA, Emmett RL, Casillas E (2005) Species composition and community structure of pelagic nekton off Oregon and Washington under variable oceanographic conditions. Mar Ecol Prog Ser 298:41-57

Clarke KR, Gorley RN (2001) Primer v5: user manual/tutorial. PRIMER-E, Plymouth, UK

Clarke KR, Warwick RM (2001) Change in marine communities: an approach to statistical analysis and interpretation. 2nd edn, PRIMER-E, Plymouth, UK

Cowen RK, Hare JA, Fahey MP (1993) Beyond hydrography: can physical processes explain larval fish assemblages in the Middle Atlantic Bight? Bull Mar Sci 53:567-587

Doyle MJ (1992) Patterns in distribution and abundance of ichthyoplankton off Washington, Oregon, and northern California (1980-1987). AFSC Proc Rep 92-14

Doyle MJ (1995) The El Niño of 1983 as reflected in the ichthyoplankton off Washington, Oregon, and northern California. Can Spec Publ Fish Aquat Sci 121:161-180

Doyle MJ, Mier KL, Busby MS, Brodeur RD (2002) Regional variations in springtime ichthyoplankton assemblages in the Northeast Pacific Ocean. Prog Oceanogr 53: $247-281$

Emmett RL, Brodeur RD, Miller TW, Pool SS, Bentley PJ, Krutzikowsky GK, McCrae J (2005) Pacific sardine (Sardinops sagax) abundance, distribution and ecological relationships in the Pacific Northwest. Calif Coop Ocean Fish Investig Rep 46:122-143

Field JG, Clarke KR, Warwick RM (1982) A practical strategy for analyzing multispecies distribution patterns. Mar Ecol Prog Ser 8:37-52 
Gómez-Gutiérrez J, Peterson WT, Miller CB (2005) Crossshelf life-stage segregation of the euphausiids off central Oregon (1970-1972). Deep-Sea Res II 52:289-315

Grantham BA, Chan F, Nielsen KJ, Fox DS, Barth JA, Huyer J, Lubchenco J, Menge BA (2004) Upwellingdriven nearshore hypoxia signals ecosystem and oceanographic changes in the northeast Pacific. Nature 429: $749-754$

Hjort J (1914) Fluctuations of the great fisheries of northern Europe viewed in the light of biological research. Rapp P-V Réun Cons Int Explor Mer 20:1-228

Houde ED (1997) Patterns and consequences of selective processes in teleost early life histories. In: Chambers RC, Trippel EA (eds) Early life history and recruitment in fish populations. Chapman \& Hall, London, p 172-196

Hunter JR, Kimbrell C (1980) Egg cannibalism in the northern anchovy, Engraulis mordax. Fish Bull US 78:811-816

Keister JE, Peterson WT (2003) Zonal and seasonal variations in zooplankton community structure off the central Oregon coast, 1998-2000. Prog Oceanogr 57:341-361

Kosro PM (2003) Enhanced southward flow over the Oregon shelf in 2002: a conduit for subarctic water. Geophys Res Lett 30:1-4

Krebs CJ (1989) Ecological methodology. Harper Collins, New York

Marancik KE, Clough LM, Hare JA (2005) Cross-shelf and seasonal variation in larval fish assemblages on the southeast United States continental shelf off the coast of Georgia. Fish Bull US 103:108-129

Matarese AC, Blood DM, Picquelle SJ, Benson JL (2003) Atlas of abundance and distribution patterns of ichthyoplankton from the northeast Pacific Ocean and Bering Sea ecosystems based on research conducted by the Alaska Fisheries Science Center (1972-1996). NOAA Prof Paper NMFS $1: 1-281$

Morgan CA, Peterson WT, Emmett RL (2003) Coastaloffshore variations in copepod community structure off the Oregon coast during the summer upwelling season. Mar Ecol Prog Ser 249:223-236

Mundy BC (1984) Yearly variation in the abundance and distribution of fish larvae in the coastal upwelling zone off Yaquina Head, OR, from June 1969-August 1972. MSc thesis, Oregon State University, Corvallis

Olivar MP (1990) Spatial patterns of ichthyoplankton distribution in relation to hydrographic features in the Northern Benguela region. Mar Biol 106:39-48

Parrish RH, Nelson CS, Bakun A (1981) Transport mechanisms and reproductive success of fishes in the California Current. Biol Oceanogr 1:175-203

Pearcy WG, Hosie MJ, Richardson SL (1977) Distribution and duration of pelagic life of larvae of Dover sole, Microstomus pacificus; rex sole, Glyptocephalus zachirus; and petrale sole, Eopsetta jordani, in waters off Oregon. Fish Bull US 75:173-183

Editorial responsibility: Kenneth Sherman (Contributing Editor), Narragansett, Rhode Island, USA
Purcell JE, Arai MN (2001) Interactions of pelagic cnidarians and ctenophores with fish: a review. Hydrobiologia 451:27-44

Richardson SL (1973) Abundance and distribution of larval fishes in waters off Oregon, May-October 1969, with special emphasis on the northern anchovy, Engraulis mordax. Fish Bull US 71:697-711

Richardson SL, Pearcy WG (1977) Coastal and oceanic larvae in an area of upwelling off Yaquina Bay, Oregon. Fish Bull US 75:125-145

Richardson SL, Laroche JL, Richardson MD (1980) Larval fish assemblages and associations in the north-east Pacific Ocean along the Oregon coast, winter-spring 1972-1975. Estuar Coast Mar Sci 11:671-699

Sabatés A (1990) Distribution pattern of larval fish populations in the Northwestern Mediterranean. Mar Ecol Prog Ser 59:75-82

Sakuma KM, Ralston S, Roberts DA (1999) Diel vertical distribution of postflexion larval Citharichthys spp. and Sebastes spp. off central California. Fish Oceanogr 8:68-76

Schwing FB, Moore C (2000) 1999-a year without summer for California or a harbinger of a climate shift? EOS Trans Am Geophys Un 81:301, 304-305

Schwing FB, Moore C, Ralston S, Sakuma KA (2000) Record coastal upwelling in the California Current in 1999. Calif Coop Ocean Fish Investig Rep 41:148-160

Shannon CE, Weaver W (1949) The mathematical theory of communication. University of Illinois Press, Urbana

Sherman K, Lasker R, Richards W, Kendall AW Jr (1983) Ichthyoplankton and fish recruitment studies in large marine ecosystems. Mar Fish Rev 45:1-25

Smith PE, Moser HG (2003) Long-term trends and variability in the larvae of Pacific sardine and associated fish species of the California Current region. Deep-Sea Res II 50: 2519-2536

Suchman CL, Brodeur RD (2005) Abundance and distribution of large medusae in surface waters of the northern California Current. Deep-Sea Res II 52:51-72

Ureña HM (1989) Distribution of eggs and larvae of some flatfishes (Pleuronectiformes) off Washington, Oregon and Northern California, 1980-83. MSc thesis, Oregon State University, Corvallis

Waldron KD (1972) Fish larvae collected from the northeastern Pacific Ocean and Puget Sound during April and May 1967. NOAA Tech Rep NMFS SSRF-663:1-16

Wheeler PA, Huyer A, Fleischbein J (2003) Cold halocline, increased nutrients and higher chlorophyll off Oregon in 2002. Geophys Res Lett 30:1-4

Wiebe PH, Burt KH, Boyd SH, Morton AW (1976) A multiple opening/closing net and environmental sampling system for sampling zooplankton. J Mar Res 34:313-326

Young JW, Davis TLO (1990) Feeding ecology of larvae of southern bluefin albacore and skipjack tunas (Pisces: Scombridae) in the eastern Indian Ocean. Mar Ecol Prog Ser 61:17-29

Submitted: July 22, 2005; Accepted: December 27, 2005 Proofs received from author(s): July 31, 2006 